\title{
Bordetella Bronchiseptica Vaccines in Pet Guinea Pigs? A Review of the Literature Gallego $\mathbf{M}^{*}$
}

Centro Veterinario Madrid Exoticos , Calle Meléndez Valdés 17, 28015, Madrid, Spain

Received: 28 February, 2017; Accepted: 07 April, 2017; Published: 17 April, 2017

*Corresponding author: Miguel Gallego Agúndez LV, Centro Veterinario Madrid Exoticos, Calle Meléndez Valdés 17, 28015, Madrid (Spain). Tel: +34 91 44608 24; E-mail: miguel.galego@gmail.com

\begin{abstract}
Bordetella bronchiseptica causes severe respiratory disease in guinea pigs and it has been associated with stillbirths, abortions, infertility and infections of the reproductive system and tympanic bullae. Disease caused by Bordetella bronchiseptica in pet guinea pigs is mostly a poor understood disease; studies about this pathogen has been conducted mostly in laboratory guinea pigs. The objective of this review is to condense published information about the disease caused by Bordetella bronchiseptica in guinea pigs for the veterinarian who attends pet guinea pigs, with special emphasis on the prevention of this disease. The author concludes that vaccination in pet guinea pigs against Bordetella bronchiseptica needs to be considered for future studies.
\end{abstract}

\section{Pathogeny}

Bordetella bronchiseptica $(\mathrm{Bb})$ is a gram-negative rod that has tropism for the ciliated respiratory epithelium. Infected pet guinea pigs can eliminate the bacteria, become asymptomatic carriers or develop the disease. The pet guinea pigs that develop the disease usually are attended at the veterinary clinic showing nasal and ocular discharge, respiratory sounds and dyspnea; the inappetence and lethargy indicate a poor prognosis. The diagnosis and treatment is based on deep nasal culture and antibiogram. Guinea pigs that have contact with the diseased guinea pig should be separated and occurrence of clinical signs should be monitorized at least until the diseased guinea pig has been cured. It is good practice to perform x-rays of the head and chest of the guinea pigs that are in contact with the diseased ones because a subclinical or early-stage disease can be detected [1-3].

Bordetella bronchiseptica causes severe respiratory disease in guinea pigs, firstly described at the end of the 19th century [4] (Figure 1). Stillbirths, abortions, infertility and infections of the reproductive system can occur if haematonous spread of the bacteria develops; also the tympanic bullae may contain purulent exudates in affected animals due to ascending infection $[1,5,6]$ (Figure 2,3). The incidence of $\mathrm{Bb}$ in a colony is usually $20 \%$ lower in laboratory animals if no action is taken, but this incidence is unknown in guinea pigs [7]. Disease caused by $\mathrm{Bb}$ in pet guinea pigs is mostly a poor understood disease because what is known of the disease is mostly by studies conducted in laboratory guinea pigs, and not in pet guinea pigs [1-3].

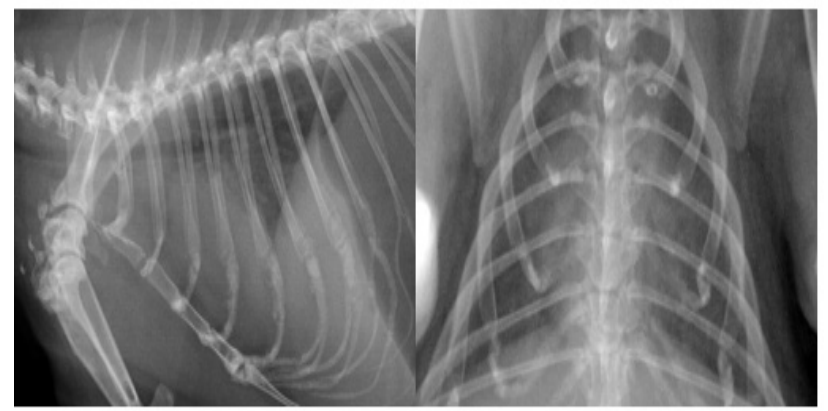

Figure 1: Laterolateral and ventrodorsal views of thoracic radiographs from a pet guinea pig with pneumonia. Necropsy tissues were submitted, the pulmonary interstitium presented infiltration of heterophiles and macrophages and the microbiological culture was positive for Bordetella bronchiseptica.

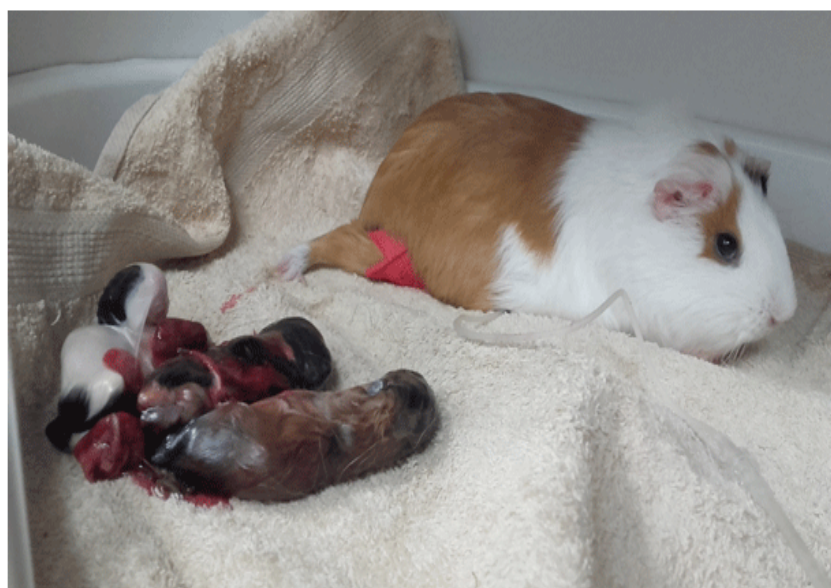

Figure 2: Stillbirths associated with Bordetella bronchiseptica infection in guinea pigs. In the case showed in this image, the deep nasal culture of the mother was positive to Bordetella bronchiseptica. 


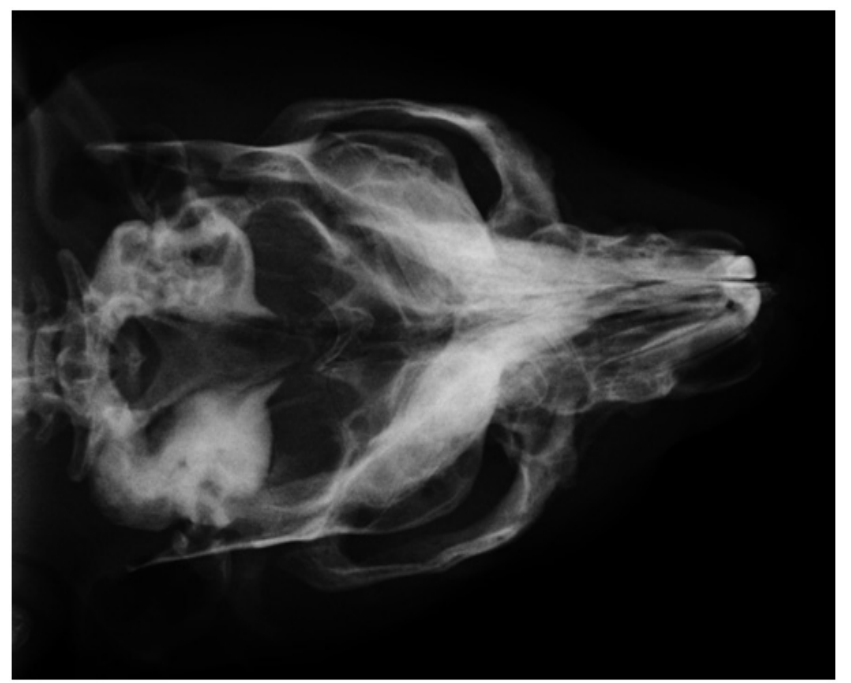

Figure 3: Dorsoventral view of a skull radiograph from a pet guinea pig with facial nerve paralysis. Severe radiodensity of the right tympanic bullae is noticed. The culture of the right tympanic bulla taken at necropsy was positive to Bordetella bronchiseptica.

Nakagawa (1969) describes the respiratory disease produced by $\mathrm{Bb}$ in guinea pigs as being low virulent with little or no mortality [7]. Others describe epizootic outbreaks with high virulence and mortality $[6,8]$. As we will see later, there are several factors that may influence these discrepancies, including the bacterial strain.

Transmission of the disease among guinea pigs requires close contact; by aerosols, nasal secretions and fomites $[5,8]$. Susceptibility is greatest in young animals and incubation period was founded to be of 5-7 days $[6,7]$.

As guinea pigs seems to be more sensitive to the pathogen than rabbits, and rabbits are frequent asymptomatic carriers and cohabitants in homes and animal shops, it is advisable not to keep them in close contact [5]. In addition, Bb has been isolated in 18 species of mammals associated with respiratory disease, the elimination of the pathogen lasts a long time in animals and there is a high percentage of asymptomatic infections [9]. For these reasons it is very possible that the disease may be quite common in pet guinea pigs, being able to act as reservoir of the bacterium.

Bordetella bronchiseptica is more pathogenic and less host specific than Bordetella pertussis and Bordetella parapertussis, both of which cause generally mild disease in man and both evolved from a common ancestor similar to $\mathrm{Bb}$, which lost virulence and gained adaptation [10]. The three bacteria are the only pathogens of the entire Bordetella genus and crossimmunity between the three is proved [11-13].

In guinea pigs, maternal immunity seems to be effective during the first four weeks, but youngs may be seropositive up to 8 weeks of age [14].

The development of the disease may be related to stressors such as inadequate diet, concurrent diseases, inadequate environmental conditions, pregnancy, overcrowding, etc., [15]. Animals recovering from the disease may be asymptomatic carriers, being immunized but also representing a source of infection for other animals [8,16-21].

\section{Vaccines}

To maintain a guinea pig colony free of $\mathrm{Bb}$, it is considered that only pathogen-free animals should be introduced [5].Vaccination seems to not prevent asymptomatic carrier status because after infection in vaccinated animals the bacteria in the upper respiratory tract of some animals are isolated, although vaccination avoids disease [11,14,17-19]. In pet guinea pigs the only feasible possibility of preventing the disease is vaccination.

Published papers on vaccine prophylaxis relate to laboratory animals and most of these papers study experimental vaccines available only for laboratory animals $[6,14,17]$. These vaccines are not an option in pet guinea-pigs, however, commercially approved dead commercial vaccines approved for use in dogs, pigs and people were successfully evaluated in laboratory animals $[18,22]$.

Vaccines best considered against $\mathrm{Bb}$ infection in other species are live attenuated by intranasal or oral application: they induce both local immunity at nasal level (IgA) and seroconversion (IgG), only one application is needed to produce longtime immunity, effective immunity is achieved rapidly (up to 72 hours) and do not provoke local reactions [23-31]. Local nasal immunity (IgA) appears to be of crucial importance in respiratory infectious diseases, particularly if Bordetella spp. is implicated $[17,23,32,33]$.

\section{Treatment}

Various treatment protocols have been proposed in pet guinea pigs based on support therapy, systemic antibiotherapy (best based on bacterial culture and sensitivity testing) and chamber nebulization with antibiotic but effectiveness of these treatments have not been proven [1-3].

\section{Conclussion}

Vaccination in pet guinea pigs against Bordetella bronchiseptica must be considered for future studies.

\section{Reference}

1. Goodman G. Rodents: respiratory and cardiovascular system disorders. In Keeble E, Meredith A, editors. BSAVA Manual of rodents and ferrets. $1^{\text {st }}$ edition. Gloucester: British Small Animal Veterinary Association. 2009;142-149

2. Riggs SM. Guinea pigs. In Mitchell MA, Tully TN, editors. Manual of exotic pet practice. $1^{\text {st }}$ edition. Missouri: Elsevier. 2009;456-473

3. Hawkins MG, Bishop CR. Disease problems of guinea pigs. In Quesenberry KE, Carpenter JW, editors. Ferrets, rabbits and rodents: clinical medicine and surgery. 3rd edition. Missouri: Elsevier. 2012;295-310

4. Tartakowsky MG. Pneumonie contagieuse des cobayes. Arch Sci Biol (St. Petersburg). 1897-1898;6:255-284. 
5. Brabb T, Newsome D, Burich A, Hanes M. Infectious diseases. In Suckow MA, Stevens KA, Wilson RP, editors. The laboratory rabbit, guinea pig, hamster and other rodents. 1st edition. London: Academic Press. 2012;1247-1268

6. Ganaway JR, Allen AR, McPherson CW. Prevention of acute Bordetella bronchiseptica pneumonia in a guinea pig colony. Lab Anim Care. $1965 ; 15: 156-62$

7. Nakagawa M, Muto T, Nakano T, Yoda H, Ando K, Isobe Y, et al. Some observations on diagnosis of Bordetella bronchiseptica infection in guinea pigs. Exp Anim. 1969;18:105-116

8. Woode GN, McLeod N. Control of acute Bordetella bronchiseptica pneumonia in a guinea-pig colony. Lab Anim. 1967;1:91-94.

9. Mattoo S, Cherry JD. Molecular pathogenesis, epidemiology, and clinical manifestations of respiratory infections due to Bordetella pertussis and other Bordetella subspecies. Clin Microbiol Rev. 2005;18(2):32682

10. Parkhill J, Sebaihia M, Preston A, Murphy LD, Thomson N, Harris DE, et al. Comparative analysis of the genome sequences of Bordetella pertussis, Bordetella parpertussis and Bordetella bronchiseptica. Nat Genet. 2003;35(1):32-40

11. Goodnow RA. Biology of Bordetella bronchiseptica. Microbiol Rev. 1980;44(4):722-738

12. Vargas MH, Bazán-Perkins B, Segura P, Campos MG, Selman M, Montaño LM. Inhaled Bordetella Pertussis vaccine decreases airway responsiveness in guinea pigs. Life Sci. 1995;57(19):293-299

13. Kendrick PL, Nadolski EB, Eldering G, Baker J. Antigenic relationships of Haemophilus pertussis, the parapertussis bacillus, and Brucella bronchiseptica as shown by cross protection tests in mice. J Bacteriol. 1953;66(2):166-169

14. Nakagawa M, Yoda H, Muto T, Imaizumi K. Prophylaxis of Bordetella bronchiseptica infection in guinea pigs by vaccination. Jap J Vet Sci. 1974;36:33-42

15.Ganaway JR. Bacterial, mycoplasma, and rickettsial diseases. In Wagner JE, Manning PJ. The Biology of the guinea pig. 1st edition. New York: Academic Press. 1976;121-135

16. Rigby C. Natural infections of guinea-pigs. Lab Anim. 1976;10(2):119142

17. Shimizu T. Prophylaxis of Bordetella bronchiseptica infection in guinea pigs by intranasal vaccination with live strain ts-S34. Infect Immun. 1978;22(2):318-321

18. Stephenson EH, Trahan CJ, Ezzel JW, Mitchell WC, Abshire TG, Oland DD, et al. Efficacy of a commercial bacterin in protecting Strain 13 guinea pigs against Bordetella bronchiseptica pneumonia. Lab Anim. 1989;23(3):261-269

19. Winsser J. A study of Bordetella bronchiseptica. Proc Anim Care Panel. 1960;10:87-104

20. Yoda H, Nakagawa M, Muto T, Imaizumi K. Development of resistance to reinfection of Bordetella bronchiseptica in guinea pigs recovered from natural infection. Jap J Vet Sci. 1972.349(4):191-196
21. Mohamed A, Reddy GP, Samuel T, Mansour M, Woubit A. Clostridium difficile (Cd) in Shelter Dogs: Rationale for Screening of Pets Slated for Adoption. SOJ Vet Sci. 2016;2(1):1-6

22. Matherne CM, Steffen EK, Wagner JE. Efficacy of commercial vaccines for protecting guinea pigs against Bordetella bronchiseptica pneumonia. Lab Anim Sci. 1987;37(2):191-194

23. Davis R, Jayappa H, Abdelmagid OY, Armstrong R, Sweeney D, Lehr C. Comparison of the mucosal immune response in dogs vaccinated with either an intranasal avirulent live culture or a subcutaneous antigen extract vaccine of Bordetella bronchiseptica. Vet Ther. 2007; 8(1):3240

24. Gore T, Headley M, Laris R, Bergman JGHE, Sutton D, Horspool LJI, et al. Intranasal kennel cough vaccine protecting dogs from experimental Bordetella bronchiseptica challenge within 72 hours. Vet Rec. 2005;156(15):482-483

25. Hess TJ, Parker DS, Hassall AJ, Chiang Y. Evaluation of efficacy of oral administration of Bordetella bronchiseptica intranasal vaccine when used to protect puppies from tracheobronchitis due to $\mathrm{B}$. bronchiseptica infection. Intern J Appl Res Vet Med. 2011. 9(3):300305

26. Jacobs AA, Theelen RP, Jaspers R, Horspool LJ, Sutton D, Bergman JG, et al. Protection of dogs for 13 months against Bordetella bronchiseptica and canine parainfluenza virus with a modified live vaccine. Vet Rec. 2005;157(1):19-23

27. Jacobs AA, Bergman JG, Theelen RP, Jaspers R, Helps JM, Horspool LJ, et al. Compatibility of a bivalent modified-live vaccine against Bordetella bronchiseptica and $\mathrm{CPiV}$, and a trivalent modified-live vaccine against CPV, CDV and CAV-2. Vet Rec. 2007;160(2):41-45

28. Larson LJ, Thiel BE, Sharp P, Schultz RD. A comparative study of protective immunity provided by oral, intranasal and parenteral canine Bordetella bronchiseptica vaccines. Intern J Appl Res Vet Med. 2013;11(3):153-160

29. Sekiya K, Kawahira M, Nakase Y. Protection against experimental Bordetella bronchiseptica infection in mice by active immunization with killed vaccine. Infect Immun. 1983;41(2):598-603

30. Williams J, Laris R, Gray AW, Jacobs AA. Studies of the efficacy of a novel intranasal vaccine against feline bordetellosis. Vet Rec. 2002;150(14):439-442

31.Zhang $\mathrm{Q}$ Hu R, He H, Tang $\mathrm{X}$, Jin $\mathrm{M}$, Chen $\mathrm{H}$, et al. aroA deleted Bordetella bronchiseptica inspiring robust mucosal immune response and provide full protection against intranasal challenge. Res Vet Sci. 2013;94(1):55-61. doi: 10.1016/j.rvsc.2012.07.019

32. Renegar KB, Small PA, Boykins LG, Wright PF. Role of IgA versus IgG in the control of influenza viral infection in the murine respiratory tract. J immunol. 2004;173(3):1978-1986

33. Wolfe DN, Kirimanjeswara GS, Goebel EM, Harvill ET. Comparative role of immunoglobulin A in protective immunity against the Bordetellae. Infect Immun. 2007;75(9):4416-4422. 